\title{
Protection of a network by complete secure domination
}

Research Article

\author{
Girish V. Rajasekharaiah, Usha P. Murthy, Umesh Subramanya
}

\begin{abstract}
A complete secure dominating set of a graph $G$ is a dominating set $D \subseteq V(G)$ with the property that for each $v \in D$, there exists $F=\left\{v_{j} \mid v_{j} \in N(v) \cap(V(G)-D)\right\}$, such that for each $v_{j} \in F$, $(D-\{v\}) \cup\left\{v_{j}\right\}$ is a dominating set. The minimum cardinality of any complete secure dominating set is called the complete secure domination number of $G$ and is denoted by $\gamma_{c s d}(G)$. In this paper, the bounds for complete secure domination number for some standard graphs like grid graphs and stacked prism graphs in terms of number of vertices of $G$ are found and also the bounds for the complete secure domination number of a tree are obtained in terms of different parameters of $G$.
\end{abstract}

2010 MSC: $05 C 69$

Keywords: Domination, Secure domination, Complete secure domination

\section{Introduction}

The graphs considered here are undirected, finite, connected, without multiple edges or loops and without isolated vertices. As usual $n$ and $q$ denote the number of vertices and edges of a graph $G$. For basic graph theoretic notation and terminology we refer to [4].

A set of vertices $D$ is said to dominate the graph $G$ if for each vertex $v \in V(G)-D$, there is a vertex $u \in D$ with $v$ is adjacent to $u$. The minimum cardinality of any dominating set is called the domination number of $G$ and it is denoted by $\gamma(G)$.

A secure dominating set $X$ of a graph $G$ is a dominating set with the property that each vertex $u \in V(G)-X$ is adjacent to a vertex $v \in X$ such that $(X-\{v\}) \cup\{u\}$ is dominating set. The minimum cardinality of such a set is called the secure domination number, denoted by $\gamma_{s}(G)$.

The Cartesian graph product $G_{1} \times G_{2}$ called graph product of graphs $G_{1}=\left(V_{1}, E_{1}\right)$ and $G_{2}=\left(V_{2}, E 2\right)$ with disjoint vertex sets is the graph with the vertex set $V_{1} \times V_{2}$ and $u=\left(u_{1}, u_{2}\right)$ adjacent with $v=\left(v_{1}, v_{2}\right)$ whenever $\left[u_{1}=v_{1}\right.$ and $u_{2}$ adj $\left.v_{2}\right]$ or $\left[u_{2}=v_{2}\right.$ and $u_{1}$ adj $\left.v_{1}\right]$.

Girish V. Rajasekharaiah, (Corresponding Author), Umesh Subramanya; Department of Science and Humanities, PES University (EC Campus), Electronic City, Bengaluru, Karnataka, India (email: girishvr1@pes.edu, giridsi63@gmail.com,umeshsubbu@gmail.com).

Usha P. Murthy; Department of Mathematics, Siddaganga Institute of Technology, B.H.Road, Tumakuru Karnataka, India (email: ushapmurhty@yahoo.com). 
A friendship graph $F_{n}$ is the graph obtained by joining $n$ copies of $C_{3}$ with a common vertex. A vertex of degree one is called an end vertex and a vertex adjacent to an end vertex is called non-end vertex.

A two-dimensional grid graph $G_{m, n}$ is the Cartesian product $P_{m} \times P_{n}$ of path graphs on $m$ and $n$ vertices. A stacked prism graph is the Cartesian product of $C_{m} \times P_{n}$.

The protection of a (simple) graph $G=(V, E)$ involves placing a set (possibly empty) of guards at each vertex, and it is assumed that a guard can deal with a problem (called an attack) at any vertex in its closed neighborhood. Various strategies(i.e., rules for guard placements) have been devised, under each of which the entire graph $G$ may be considered protected. The minimum number of guards required for protection under each strategy is clearly of interest. The concept of secure domination was introduced by Cockayne et.al. [3]. Later this concept was studied extensively in [1, 2].

In social network theory, we can assume the graph nodes as message centers and its edges as transmission lines. The message is transferred through the transmission line from one message center to another. The intent of this process is to transfer the messages to all message centers with minimum number of message centers. To find all the minimum message centers, we can make use of domination and these minimum message centers are called as domination message centers. And if any of the domination message centers has an issue in sending the message, then it's neighbor message center should act as a domination message center to complete the process. This solution is illustrated in secure domination concept, the drawback in this secure domination concept is that, it is just mentioned to select their neighbors as domination message centers, but not mentioned about which particular neighbor has to be chosen as a domination message center to achieve the objective or to complete the process. So, to overcome these problems we introduce a new concept called as complete secure domination with which one can select a neighbor as domination message centers. So that, we would be able to provide an analogy to retrieve a more efficient and robust domination message centers to solve the above mentioned issue and to transfer messages in a more faster and constructive manner.

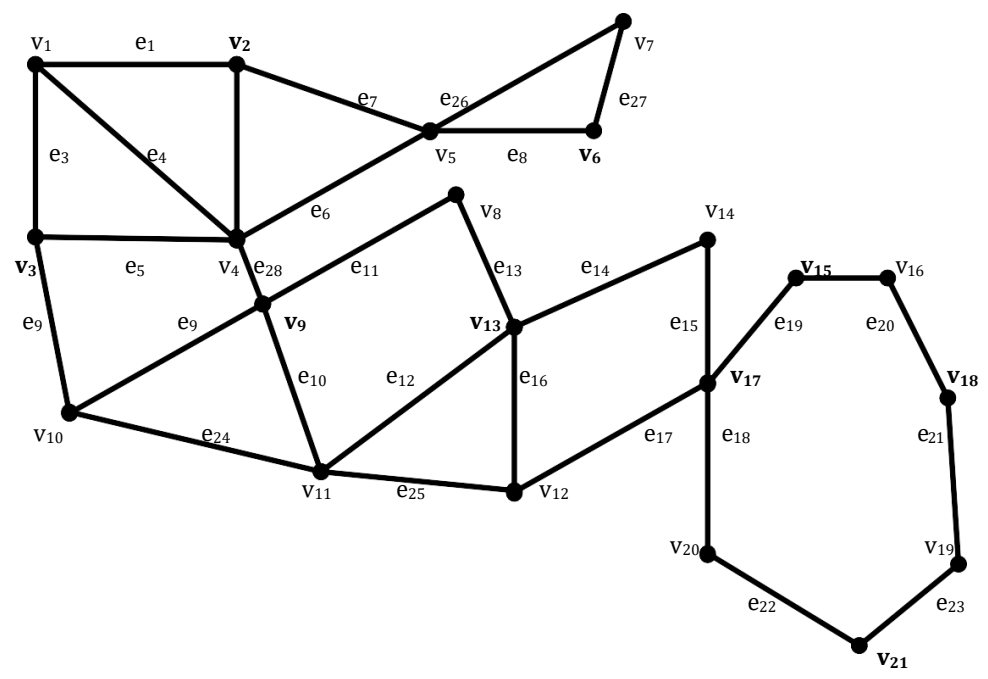

Figure 1. Example for complete secure domination

Minimum dominating set $D=\left\{v_{5}, v_{4}, v_{11}, v_{13}, v_{18}, v_{17}, v_{21}\right\}$.

Minimum secure dominating set $C=\left\{v_{6}, v_{2}, v_{3}, v_{9}, v_{13}, v_{18}, v_{17}, v_{21}\right\}$.

Minimum complete secure dominating set $F=\left\{v_{6}, v_{2}, v_{3}, v_{9}, v_{13}, v_{18}, v_{17}, v_{21}, v_{15}\right\}$.

$$
|D|=7,|C|=8,|F|=9
$$


A complete secure dominating set of a graph $G$ is a dominating set $D \subseteq V(G)$ with the property that for each $v \in D$, there exists $F=\left\{v_{j} \mid v_{j} \in N(v) \cap(V(G)-D)\right\}$, such that for each $v_{j} \in F,(D-\{v\}) \cup\left\{v_{j}\right\}$ is a dominating set. The minimum cardinality of any complete secure dominating set is called the complete secure domination number of $G$ and is denoted by $\gamma_{c s d}(G)$.

\section{Complete secure domination for standard graphs}

Theorem 2.1. For any path $P_{n}, \gamma_{c s d}\left(P_{n}\right)=\left\lceil\frac{n}{2}\right\rceil$.

Theorem 2.2. For any wheel graph $W_{n}, \gamma_{c s d}\left(W_{n}\right)=\left\lceil\frac{n}{3}\right\rceil$.

Theorem 2.3. For any cycle $C_{n}, \gamma_{c s d}\left(C_{n}\right)=\left\lceil\frac{n}{2}\right\rceil$.

Theorem 2.4. For any friendship graph $F_{n}$ with $n$ vertices, $\gamma_{c s d}\left(F_{n}\right)=\left\lceil\frac{n}{3}\right\rceil$.

\section{Main results}

Theorem 3.1. For any graph $G=P_{2} \times P_{n}, \gamma_{c s d}(G)=n, n \geq 2$.

Proof. Let $V\left(P_{2} \times P_{n}\right)=\left\{\left(u_{i}, v_{s}\right), s=1,2,3, \ldots, n\right\}_{i=1}^{i=2}$ be the vertices of first and second row, respectively. We consider the following cases.

Case 1. Suppose $n$ is even.

Let $A=\left\{\left(u_{1}, v_{s}\right), s=2 p, 1 \leq p \leq \frac{n}{2}\right\}$ and $B=\left\{\left(u_{2}, v_{s}\right), s=2 p-1,1 \leq p \leq \frac{n}{2}\right\}$ with $|A|=\frac{n}{2}$ and $|B|=\frac{n}{2}$. Let $D=A \cup B$, every neighborhood vertex of $V(G)-D$ is in $D, D$ is the complete secure dominating set of $G$. Hence $\gamma_{c s d}(G) \leq|D|$. If $\gamma_{c s d}(G)<|D|$, then there exits at least one vertex say $v_{j} \in V(G),\left(D-\left\{v_{i}\right\}\right) \cup\left\{v_{j}\right\}, v_{i} \in D$ is not a dominating set. Therefore $D$ is the $\gamma_{c s d}$-set of $G$. Hence $\gamma_{c s d}(G)=|D|=|A|+|B|=\frac{n}{2}+\frac{n}{2}=n$.

Case 2. Suppose $n$ is odd.

Let $A=\left\{\left(u_{1}, v_{s}\right), s=2 p, 1 \leq p \leq \frac{n-1}{2}\right\}$ and $B=\left\{\left(u_{2}, v_{s}\right), s=2 p-1,1 \leq p \leq \frac{n+1}{2}\right\}$ with $|A|=\frac{n-1}{2}$ and $|B|=\frac{n+1}{2}$. Let $D=A \cup B$, every neighborhood vertex of $V(G)-D$ is in $D, D$ is the complete secure dominating set of $G$. Hence $\gamma_{c s d}(G) \leq|D|$. If $\gamma_{c s d}(G)<|D|$, then there exits at least one vertex say $v_{j} \in V(G),\left(D-\left\{v_{i}\right\}\right) \cup\left\{v_{j}\right\}, v_{i} \in D$ is not a dominating set. Therefore $D$ is the $\gamma_{c s d}$-set of $G$. Hence $\gamma_{c s d}(G)=|D|=|A|+|B|=\frac{n-1}{2}+\frac{n+1}{2}=n$.

The proof is complete.

Theorem 3.2. For any graph $G=P_{3} \times P_{n}, n \geq 2$,

$$
\gamma_{c s d}(G)= \begin{cases}\frac{3 n}{2} & n \text { is even } \\ \frac{3 n-1}{2} & n \text { is odd. }\end{cases}
$$

Proof. Let $V\left(P_{3} \times P_{n}\right)=\left\{\left(u_{i}, v_{s}\right), s=1,2,3, \ldots, n\right\}_{i=1}^{i=3}$ be the vertices of first, second and thrid row, respectively. We consider the following cases.

Case 1. Suppose $n$ is even.

Let $A=\left\{\left(u_{r}, v_{s}\right), s=2 p, 1 \leq p \leq \frac{n}{2}, r=1,3\right\}$ and $B=\left\{\left(u_{r}, v_{s}\right), s=2 p-1,1 \leq p \leq \frac{n}{2}, r=2\right\}$ with $|A|=2 * \frac{n}{2}$ and $|B|=\frac{n}{2}$. Let $D=A \cup B$, every neighborhood vertex of $V(G)-D$ is in $D, D$ is the complete secure dominating set of $G$. Hence $\gamma_{c s d}(G) \leq|D|$. If $\gamma_{c s d}(G)<|D|$, then there exits at least one vertex say $v_{j} \in V(G),\left(D-\left\{v_{i}\right\}\right) \cup\left\{v_{j}\right\}, v_{i} \in D$ is not a dominating set. Therefore $D$ is the $\gamma_{c s d}$-set of $G$. Hence $\gamma_{c s d}(G)=|D|=|A|+|B|=n+\frac{n}{2}=\frac{3 n}{2}$. 
Case 2. Suppose $n$ is odd.

Let $A=\left\{\left(u_{r}, v_{s}\right), s=2 p, 1 \leq p \leq \frac{n-1}{2}, r=1,3\right\}$ and $B=\left\{\left(u_{r}, v_{s}\right), s=2 p-1,1 \leq p \leq \frac{n+1}{2}, r=\right.$ $2\}$ with $|A|=2\left(\frac{n-1}{2}\right)$ and $|B|=\frac{n+1}{2}$. Let $D=A \cup B$, every neighborhood vertex of $V(G)-D$ is in $D, D$ is the complete secure dominating set of $G$. Hence $\gamma_{c s d}(G) \leq|D|$. If $\gamma_{c s d}(G)<|D|$, then there exits at least one vertex say $v_{j} \in V(G),\left(D-\left\{v_{i}\right\}\right) \cup\left\{v_{j}\right\}, v_{i} \in D$ is not a dominating set. Therefore $D$ is the $\gamma_{c s d}$-set of $G$. Hence $\gamma_{c s d}(G)=|D|=|A|+|B|=n-1+\frac{n+1}{2}=\frac{3 n-1}{2}$.

The proof is complete.

Theorem 3.3. For any graph $G=P_{4} \times P_{n}, \gamma_{c s d}(G)=2 n, n \geq 2$.

Proof. Let $V\left(P_{4} \times P_{n}\right)=\left\{\left(u_{i}, v_{s}\right), s=1,2,3, \ldots, n\right\}_{i=1}^{i=4}$ be the vertices of first, second, thrid and fourth row, respectively. We consider the following cases.

Case 1. Suppose $n$ is even.

Let $A=\left\{\left(u_{r}, v_{s}\right), s=2 p, 1 \leq p \leq \frac{n}{2}, r=1,3\right\}$ and $B=\left\{\left(u_{r}, v_{s}\right), s=2 p-1,1 \leq p \leq \frac{n}{2}, r=2,4\right\}$ with $|A|=2 * \frac{n}{2}$ and $|B|=2 * \frac{n}{2}$. Let $D=A \cup B$, every neighborhood vertex of $V(G)-D$ is in $D, D$ is the complete secure dominating set of $G$. Hence $\gamma_{c s d}(G) \leq|D|$. If $\gamma_{c s d}(G)<|D|$, then there exits at least one vertex say $v_{j} \in V(G),\left(D-\left\{v_{i}\right\}\right) \cup\left\{v_{j}\right\}, v_{i} \in D$ is not a dominating set. Therefore $D$ is the $\gamma_{c s d}$-set of $G$. Hence $\gamma_{c s d}(G)=|D|=|A|+|B|=n+n=2 n$.

Case 2. Suppose $n$ is odd.

Let $A=\left\{\left(u_{r}, v_{s}\right), s=2 p, 1 \leq p \leq \frac{n-1}{2}, r=1,3\right\}$ and $B=\left\{\left(u_{r}, v_{s}\right), s=2 p-1,1 \leq p \leq \frac{n+1}{2}, r=\right.$ $2,4\}$ with $|A|=2\left(\frac{n-1}{2}\right)$ and $|B|=2\left(\frac{n+1}{2}\right)$. Let $D=A \cup B$, every neighborhood vertex of $V(G)-D$ is in $D, D$ is the complete secure dominating set of $G$. Hence $\gamma_{c s d}(G) \leq|D|$. If $\gamma_{c s d}(G)<|D|$, then there exits at least one vertex say $v_{j} \in V(G),\left(D-\left\{v_{i}\right\}\right) \cup\left\{v_{j}\right\}, v_{i} \in D$ is not a dominating set. Therefore $D$ is the $\gamma_{c s d}$-set of $G$. Hence $\gamma_{c s d}(G)=|D|=|A|+|B|=n-1+n+1=2 n$.

The proof is complete.

Theorem 3.4. For any graph $G=P_{5} \times P_{n}, n \geq 2$,

$$
\gamma_{c s d}(G)= \begin{cases}\frac{5 n}{2} & n \text { is even } \\ \frac{5 n-1}{2} & n \text { is odd. }\end{cases}
$$

Proof. Let $V\left(P_{5} \times P_{n}\right)=\left\{\left(u_{i}, v_{s}\right), s=1,2,3, \ldots, n\right\}_{i=1}^{i=5}$ be the vertices of first, second, third, fourth and fifth row, respectively. We consider the following cases.

Case 1. Suppose $n$ is even.

Let $A=\left\{\left(u_{r}, v_{s}\right), s=2 p, 1 \leq p \leq \frac{n}{2}, r=1,3,5\right\}$ and $B=\left\{\left(u_{r}, v_{s}\right), s=2 p-1,1 \leq p \leq \frac{n}{2}, r=\right.$ $2,4\}$ with $|A|=3 * \frac{n}{2}$ and $|B|=2 * \frac{n}{2}$. Let $D=A \cup B$, every neighborhood vertex of $\bar{V}(G)-D$ is in $D, D$ is the complete secure dominating set of $G$. Hence $\gamma_{c s d}(G) \leq|D|$. Hence $\gamma_{c s d}(G) \leq|D|$. If $\gamma_{c s d}(G)<|D|$, then there exits at least one vertex say $v_{j} \in V(G),\left(D-\left\{v_{i}\right\}\right) \cup\left\{v_{j}\right\}, v_{i} \in D$ is not a dominating set. Therefore $D$ is the $\gamma_{c s d}$-set of $G$. Hence $\gamma_{c s d}(G)=|D|=|A|+|B|=\frac{3 n}{2}+n=\frac{5 n}{2}$.

Case 2. Suppose $n$ is odd.

Let $A=\left\{\left(u_{r}, v_{s}\right), s=2 p, 1 \leq p \leq \frac{n-1}{2}, r=1,3,5\right\}$ and $B=\left\{\left(u_{r}, v_{s}\right), s=2 p-1,1 \leq p \leq\right.$ $\left.\frac{n+1}{2}, r=2,4\right\}$ with $|A|=3\left(\frac{n-1}{2}\right)$ and $|B|=2\left(\frac{n+1}{2}\right)$. The set $D=A \cup B$ is the $\gamma_{c s d}$-set of $G$. Hence $\gamma_{c s d}(G)=|D|=|A|+|B|=3\left(\frac{n-1}{2}\right)+n+1=\frac{5 n-1}{2}$.

The proof is complete. 
Theorem 3.5. For any graph $G=P_{m} \times P_{n}, m, n \geq 2$,

$$
\gamma_{c s d}(G)= \begin{cases}\frac{m n}{2} & m \text { is even } \\ \frac{m n}{2} & m \text { is odd, } n \text { is even } \\ \frac{m n-1}{2} & m \text { is odd }, n \text { is odd. }\end{cases}
$$

Proof. Let $V\left(P_{m} \times P_{n}\right)=\left\{\left(u_{r}, v_{s}\right), s=1,2,3, \ldots, n\right\}_{r=1}^{r=m}$ be the vertices of first, second, third, ..., $m^{\text {th }}$ row, respectively. We consider the following cases.

Case 1. Suppose $m$ is even and $n$ is even.

Let $A=\left\{\left(u_{r}, v_{s}\right), r=2 p-1,1 \leq p \leq \frac{m}{2}, s=2 q, 1 \leq q \leq \frac{n}{2}\right\}$ and $B=\left\{\left(u_{r}, v_{s}\right), r=2 p, 1 \leq p \leq\right.$ $\left.\frac{m}{2}, s=2 q-1,1 \leq q \leq \frac{n}{2}\right\}$ with $|A|=\frac{m n}{4}$ and $|B|=\frac{m n}{4}$. Let $D=A \cup B$, every neighborhood vertex of $V(G)-D$ is in $D, D$ is the complete secure dominating set of $G$. Hence $\gamma_{c s d}(G) \leq|D|$. If $\gamma_{c s d}(G)<|D|$, then there exits at least one vertex say $v_{j} \in V(G),\left(D-\left\{v_{i}\right\}\right) \cup\left\{v_{j}\right\}, v_{i} \in D$ is not a dominating set. Therefore $D$ is the $\gamma_{c s d}$-set of $G$. Hence $\gamma_{c s d}(G)=|D|=|A|+|B|=$ $\frac{m n}{4}+\frac{m n}{4}=\frac{m n}{2}$.

Case 2. Suppose $m$ is even and $n$ is odd.

Let $A=\left\{\left(u_{r}, v_{s}\right), r=2 p-1,1 \leq p \leq \frac{m}{2}, s=2 q, 1 \leq q \leq \frac{n-1}{2}\right\}$ and $B=\left\{\left(u_{r}, v_{s}\right), r=2 p, 1 \leq\right.$ $\left.p \leq \frac{m}{2}, s=2 q-1,1 \leq q \leq \frac{n+1}{2}\right\}$ with $|A|=\frac{m(n-1)}{4}$ and $|B|=\frac{m(n+1)}{4}$. Let $D=A \cup B$, every neighborhood vertex of $V(G)-D$ is in $D, D$ is the complete secure dominating set of $G$. Hence $\gamma_{c s d}(G) \leq|D|$. If $\gamma_{c s d}(G)<|D|$, then there exits at least one vertex say $v_{j} \in V(G)$, $\left(D-\left\{v_{i}\right\}\right) \cup\left\{v_{j}\right\}, v_{i} \in D$ is not a dominating set. Therefore $D$ is the $\gamma_{c s d}$-set of $G$. Hence $\gamma_{c s d}(G)=|D|=|A|+|B|=\frac{m(n-1)}{4}+\frac{m(n+1)}{4}=\frac{m n}{2}$.

Case 3. Suppose $m$ is odd and $n$ is even.

Let $A=\left\{\left(u_{r}, v_{s}\right), r=2 p-1,1 \leq p \leq \frac{m+1}{2}, s=2 q, 1 \leq q \leq \frac{n}{2}\right\}$ and $B=\left\{\left(u_{r}, v_{s}\right), r=2 p, 1 \leq\right.$ $\left.p \leq \frac{m-1}{2}, s=2 q-1,1 \leq q \leq \frac{n}{2}\right\}$ with $|A|=\frac{(m+1) n}{4}$ and $|B|=\frac{(m-1) n}{4}$. Let $D=A \cup B$, every neighborhood vertex of $V(G)-D$ is in $D, D$ is the complete secure dominating set of $G$. Hence $\gamma_{c s d}(G) \leq|D|$. If $\gamma_{c s d}(G)<|D|$, then there exits at least one vertex say $v_{j} \in V(G)$, $\left(D-\left\{v_{i}\right\}\right) \cup\left\{v_{j}\right\}, v_{i} \in D$ is not a dominating set. Therefore $D$ is the $\gamma_{c s d}$-set of $G$. Hence $\gamma_{c s d}(G)=|D|=|A|+|B|=\frac{(m+1) n}{4}+\frac{(m-1) n}{4}=\frac{m n}{2}$.

Case 4. Suppose $m$ is odd and $n$ is odd.

Let $A=\left\{\left(u_{r}, v_{s}\right), r=2 p-1,1 \leq p \leq \frac{m+1}{2}, s=2 q, 1 \leq q \leq \frac{n-1}{2}\right\}$ and $B=\left\{\left(u_{r}, v_{s}\right), r=2 p, 1 \leq\right.$ $\left.p \leq \frac{m-1}{2}, s=2 q-1,1 \leq q \leq \frac{n+1}{2}\right\}$ with $|A|=\frac{(m+1)(n-1)}{4}$ and $|B|=\frac{(m-1)(n+1)}{4}$. Let $D=A \cup B$, every neighborhood vertex of $V(G)-D$ is in $D, D^{4}$ is the complete secure dominating set of $G$. Hence $\gamma_{c s d}(G) \leq|D|$. If $\gamma_{c s d}(G)<|D|$, then there exits at least one vertex say $v_{j} \in V(G)$, $\left(D-\left\{v_{i}\right\}\right) \cup\left\{v_{j}\right\}, v_{i} \in D$ is not a dominating set. Therefore $D$ is the $\gamma_{c s d}$-set of $G$. Hence $\gamma_{c s d}(G)=|D|=|A|+|B|=\frac{(m+1)(n-1)}{4}+\frac{(m-1)(n+1)}{4}=\frac{m n-1}{2}$.

The proof is complete.

Theorem 3.6. For any graph, $G=C_{3} \times P_{n}, \gamma_{c s d}(G)=n, n \geq 3$.

Proof. Let $V\left(C_{3} \times C_{n}\right)=\left\{\left(u_{i}, v_{s}\right), s=1,2,3, \ldots, n\right\}_{i=1}^{i=3}$ be the vertices of first, second and third row, respectively. The set $A=\left\{\left(u_{1}, v_{s}\right), s=1,2,3, \ldots, n\right\}$ with $|A|=n$. Since $A$ is the $\gamma$-set of $G$ and therefore $A$ is the $\gamma_{c s d}$-set of $G$. Hence $\gamma_{c s d}(G)=n$.

Theorem 3.7. For any graph, $G=C_{4} \times C_{n}, \gamma_{c s d}(G)=2 n, n \geq 3$. 
Proof. Let $V\left(C_{3} \times C_{n}\right)=\left\{\left(u_{i}, v_{s}\right), s=1,2,3, \ldots, n\right\}_{r=1}^{i=4}$ be the vertices of first, second, third and fourth row, respectively. The set $D=\left\{\left(u_{1}, v_{s}\right),\left(u_{3}, v_{s}\right), s=1,2,3, \ldots, n\right\}$ with $|D|=2 n$. Since every neighborhood vertex of $V(G)-D$ is in $D, D$ is the complete secure dominating set of $G$. Hence $\gamma_{c s d}(G) \leq$ $|D|$. If $\gamma_{c s d}(G)<|D|$, then there exits at least one vertex say $v_{j} \in V(G),\left(D-\left\{v_{i}\right\}\right) \cup\left\{v_{j}\right\}, v_{i} \in D$ is not a dominating set. Therefore $D$ is the $\gamma_{c s d}$-set of $G$. Hence $\gamma_{c s d}(G)=|D|=2 n$.

Theorem 3.8. For any graph $G=C_{m} \times P_{n}, m \neq 3, n \geq 3$,

$$
\gamma_{c s d}(G)= \begin{cases}\frac{m n}{2} & m \text { is even or odd and } n \text { is even } \\ \frac{m n}{2} & m \text { is even }, n \text { is odd. } \\ \frac{m n-1}{2} & m \text { is odd }, n \text { is odd. }\end{cases}
$$

Proof. Let $V\left(C_{m} \times C_{n}\right)=\left\{\left(u_{r}, u_{s}\right), s=1,2,3, \ldots, n\right\}_{r=1}^{r=m}$ be the vertices of first, second, third, ... $m^{\text {th }}$ row, respectively. We consider the following cases.

Case 1. Suppose $m$ is even and $n$ is even.

Let $A=\left\{\left(u_{r}, v_{s}\right), r=2 p-1,1 \leq p \leq \frac{m}{2}, s=2 q, 1 \leq q \leq \frac{n}{2}\right\}$ and $B=\left\{\left(u_{r}, v_{s}\right), r=2 p, 1 \leq p \leq\right.$ $\left.\frac{m}{2}, s=2 q-1,1 \leq q \leq \frac{n}{2}\right\}$ with $|A|=\frac{m n}{4}$ and $|B|=\frac{m n}{4}$. Let $D=A \cup B$, every neighborhood vertex of $V(G)-D$ is in $D, D$ is the complete secure dominating set of $G$. Hence $\gamma_{c s d}(G) \leq|D|$. If $\gamma_{c s d}(G)<|D|$, then there exits at least one vertex say $v_{j} \in V(G),\left(D-\left\{v_{i}\right\}\right) \cup\left\{v_{j}\right\}, v_{i} \in D$ is not a dominating set. Therefore $D$ is the $\gamma_{c s d}$-set of $G$. Hence $\gamma_{c s d}(G)=|D|=|A|+|B|=$ $\frac{m n}{4}+\frac{m n}{4}=\frac{m n}{2}$.

Case 2. Suppose $m$ is even and $n$ is odd.

Let $A=\left\{\left(u_{r}, v_{s}\right), r=2 p-1,1 \leq p \leq \frac{m}{2}, s=2 q, 1 \leq q \leq \frac{n-1}{2}\right\}$ and $B=\left\{\left(u_{r}, v_{s}\right), r=2 p, 1 \leq\right.$ $\left.p \leq \frac{m}{2}, s=2 q-1,1 \leq q \leq \frac{n+1}{2}\right\}$ with $|A|=\frac{m(n-1)}{4}$ and $|B|=\frac{m(n+1)}{4}$. Let $D=A \cup B$, every neighborhood vertex of $V(G)-D$ is in $D, D$ is the complete secure dominating set of $P_{m} \times P_{n}$. Hence $\gamma_{c s d}(G) \leq|D|$. If $\gamma_{c s d}(G)<|D|$, then there exits at least one vertex say $v_{j} \in V(G)$, $\left(D-\left\{v_{i}\right\}\right) \cup\left\{v_{j}\right\}, v_{i} \in D$ is not a dominating set. Therefore $D$ is the $\gamma_{c s d}$-set of $G$. Hence $\gamma_{c s d}(G)=|D|=|A|+|B|=\frac{m(n-1)}{4}+\frac{m(n+1)}{4}=\frac{m n}{2}$.

Case 3. Suppose $m$ is odd and $n$ is even.

Let $A=\left\{\left(u_{r}, v_{s}\right), r=2 p-1,1 \leq p \leq \frac{m+1}{2}, s=2 q, 1 \leq q \leq \frac{n}{2}\right\}$ and $B=\left\{\left(u_{r}, v_{s}\right), r=2 p, 1 \leq\right.$ $\left.p \leq \frac{m-1}{2}, s=2 q-1,1 \leq q \leq \frac{n}{2}\right\}$ with $|A|=\frac{(m+1) n}{4}$ and $|B|=\frac{(m-1) n}{4}$. Let $D=A \cup B$, every neighborhood vertex of $V(G)-D$ is in $D, D$ is the complete secure dominating set of $G$. Hence $\gamma_{c s d}(G) \leq|D|$. If $\gamma_{c s d}(G)<|D|$, then there exits at least one vertex say $v_{j} \in V(G)$, $\left(D-\left\{v_{i}\right\}\right) \cup\left\{v_{j}\right\}, v_{i} \in D$ is not a dominating set. Therefore $D$ is the $\gamma_{c s d}$-set of $G$. Hence $\gamma_{c s d}(G)=|D|=|A|+|B|=\frac{(m+1) n}{4}+\frac{(m-1) n}{4}=\frac{m n}{2}$.

Case 4. Suppose $m$ is odd and $n$ is odd.

Let $A=\left\{\left(u_{r}, v_{s}\right), r=2 p-1,1 \leq p \leq \frac{m+1}{2}, s=2 q, 1 \leq q \leq \frac{n-1}{2}\right\}$ and $B=\left\{\left(u_{r}, v_{s}\right), r=2 p, 1 \leq\right.$ $\left.p \leq \frac{m-1}{2}, s=2 q-1,1 \leq q \leq \frac{n+1}{2}\right\}$ with $|A|=\frac{(m+1)(n-1)}{4}$ and $|B|=\frac{(m-1)(n+1)}{4}$. Let $D=A \cup B$, every neighborhood vertex of $V(G)-D$ is in $D, D$ is the complete secure dominating set of $G$. Hence $\gamma_{c s d}(G) \leq|D|$. If $\gamma_{c s d}(G)<|D|$, then there exits at least one vertex say $v_{j} \in V\left(P_{m} \times P_{n}\right)$, $\left(D-\left\{v_{i}\right\}\right) \cup\left\{v_{j}\right\}, v_{i} \in D$ is not a dominating set. Therefore $D$ is the $\gamma_{c s d}$-set of $G$. Hence $\gamma_{c s d}(G)=|D|=|A|+|B|=\frac{(m+1)(n-1)}{4}+\frac{(m-1)(n+1)}{4}=\frac{m n-1}{2}$.

The proof is complete.

Theorem 3.9. For any graph $G$, if every non-end vertex is adjacent with an end-vertex then, $\gamma_{c s d}(G)=p$, where $p$ is the number of end-vertices of $G$. 
Proof. Let $A=\left\{v_{i} \in V(G) \mid d\left(v_{i}\right)=1\right\}, B=\left\{v_{j} \in V(G)-A \mid v_{j} \in N\left(v_{i}\right)\right\}$ and $C=\left(A-\left\{v_{r}\right\}\right) \cup$ $\left\{v_{j}\right\}, v_{r} \in A, v_{j} \in B \cap N\left(v_{i}\right)$. Let $D=A$ or $C$. Now for each $v \in D$, there exists $F=\left\{v_{j} \mid v_{j} \in\right.$ $N(v) \cap V(G)-D\}$, such that for each $v_{j} \in F,(D-\{v\}) \cup\left\{v_{j}\right\}$ is a dominating set. Hence $D$ is a complete secure dominating set of $G$. Now we show that there exists no other minimum complete secure dominating set other than $D$. If suppose $F \neq D$ is a minimum complete secure domination set of $G$ with $|F|<|D|$, then either $F$ is not a dominating set or for atleast one vertex $v \in F$ there exists $v_{j} \in N(v)$ such that $(F-\{v\}) \cup\left\{v_{j}\right\}$ is not a dominating set of $G$. Hence $\gamma_{c s d}(G)=|D|=p$.

Corollary 3.10. For any graph $G$, if every non-end vertex is adjacent with an end-vertex then, $\gamma_{c s d}(G)=$ $\gamma_{n s}(G)$, where $\gamma_{n s}(G)$ is the nonsplit domination number of $G$.

Proof. If every non-end vertex is adjacent with an end-vertex then, $\gamma_{n s}(G)=p, p$ is the number of end vertices of $G$, then by Theorem 3.9, the result follows.

Theorem 3.11. For any tree $T$ with $n$ vertices, $\gamma_{c s d}(T) \leq p+\left\lceil\frac{n-2 p}{2}\right\rceil, p$ is the number of end vertices of $T$.

Proof. Let $A=\left\{v_{i} \in V(T) \mid d\left(v_{i}\right)=1\right\}$ and $B=\left\{v_{j} \in V(T)-A \mid v_{j} \in N\left(v_{i}\right)\right\}$. We consider the following cases.

Case 1. If every non-end vertex of $T$ is adjacent to an end-vertex then by Theorem $3.9, \gamma_{c s d}(T)=p$.

Case 2. If atleast one vertex say $v_{j} \in V(G), v_{j}$ is not adjacent to an end-vertex say $v_{i} \in A$, then the graph $T-(A \cup B)$ will be a tree with $n-2 p$ vertices and partition the vertex set of $T-(A \cup B)$ into two disjoint sets say $R$ and $S$ such that $v_{i} \in R$ and $v_{j} \in S, v_{i} \in N\left(v_{j}\right)$ and $|R| \geq|S|$. Now consider the set $D=A \cup R$, then for each $v \in D$, there exists $F=\left\{v_{j} \mid v_{j} \in N(v) \cap V(T)-D\right\}$, such that for each $v_{j} \in F,(D-\{v\}) \cup\left\{v_{j}\right\}$ is a dominating set. Therefore $F$ is the complete secure dominating set of $G$. Hence $\gamma_{c s d}(T) \leq F=p+\left\lceil\frac{n-2 p}{2}\right\rceil$.

Theorem 3.12. For any graph $G=K_{2} \times K_{1, n-1}, \gamma_{c s d}(G)=n, n \geq 3$.

Proof. Let $V\left(K_{2}\right)=\left\{u_{1}, u_{2}\right\}$ and $V\left(K_{1, n-1}\right)=\left\{v_{1}, v_{2}, v_{3}, \ldots, v_{n}\right\}$ with $d\left(v_{1}\right)=n-1$. Let $V(G)=$ $V\left(K_{2} \times K_{1, n-1}\right)=A \cup B, A=\left\{\left(u_{i}, v_{j}\right) \in V(G) / d\left(\left(u_{i}, v_{j}\right)\right)=n\right\}, B=\left\{\left(u_{r}, v_{s}\right) \in V(G)-A\right\}$ with $|A|=2,|B|=2(n-1)$. Partition the vertex of $B$ into disjoint vertex sets, $B=H \cup R$ such that $\left(u_{i}, v_{j}\right) \in$ $N\left(\left(u_{r}, v_{s}\right)\right),\left(u_{i}, v_{j}\right) \in H,\left(u_{r}, v_{s}\right) \in R$ with $|H|=|R|=n-1$. Any minimum dominating set $D$ of $G$ has to contain two vertices say $F=\left\{\left(u_{1}, v_{1}\right),\left(u_{2}, v_{1}\right)\right\}$ where $\left\{\left(u_{1}, v_{1}\right),\left(u_{2}, v_{2}\right)\right\} \in A$. Suppose $D$ is a complete secure dominating set, then the induced graph $M=\left\langle V(G)-\left(\left\{\left(u_{1}, v_{1}\right)\right\}\right.\right.$ or $\left.\left.\left\{\left(u_{2}, v_{1}\right)\right\}\right)\right\rangle$ will contains $n-1$ support vertices and by Theorem 3.9, $\gamma_{c s d}(M)=n-1$ and if $\gamma_{c s d}(G)=n-1$, then there exists at least one vertex say $\left(u_{i}, v_{j}\right) \in V(G)$ which is not dominated by $\left(D-\left\{\left(u_{m}, v_{r}\right)\right\} \cup\left(u_{i}, v_{j}\right),\left(u_{m}, v_{r}\right) \in D \cup N\left(\left(u_{i}, v_{j}\right)\right)\right.$. Therefore $\gamma_{c s d}>n-1$. Since each vertex in $B$ is of degree 2 and belongs to neighborhood of $D$, therefore the dominating set has to contain the vertices of $H$ or $R$ together with any vertex of $A$. Now consider the set $K=H \cup\left\{\left(u_{1}, v_{1}\right)\right\}$, for each vertex $\left(u_{i}, v_{j}\right) \in K$, there exists $F=\left\{\left(u_{r}, v_{s}\right) /\left(u_{r}, v_{s}\right) \in\right.$ $\left.N\left(\left(u_{i}, v_{j}\right)\right) \cap(V(G)-D)\right\}$, such that for each $\left(u_{r}, v_{s}\right) \in F,\left(D-\left\{\left(u_{i}, v_{j}\right)\right\}\right) \cup\left\{\left(u_{r}, v_{s}\right)\right\}$ is a dominating set. Hence $K$ is a complete secure dominating set, $\gamma_{c s d}=|K|=n$.

Definition 3.13. Planar honeycomb graphs are the graphs obtained by connecting some equal regular hexagons such that any two adjacent hexagons have one edge in common. The planar honeycomb lattice is also called benzoid and it is denoted by $B_{n}$.

Theorem 3.14. For any planar honeycomb graph $B_{n}$ with $n$ vertices, $n \geq 6$,

$$
\gamma_{c s d}\left(B_{n}\right)=\left\lceil\frac{n}{2}\right\rceil
$$

Proof. Let $V\left(B_{n}\right)=\left\{u_{1 i}, u_{2 i}, u_{3 i}, \ldots, u_{p i}, i=1,2,3, \ldots, q\right\}$ denotes the first, second, third, fourth, .., $p^{\text {th }}$ row, respectively. We consider the following cases.

Case 1 . When $n=6$.

In this case the graph $B_{6}=C_{6}$. The result follows from Theorem 2(iii). 


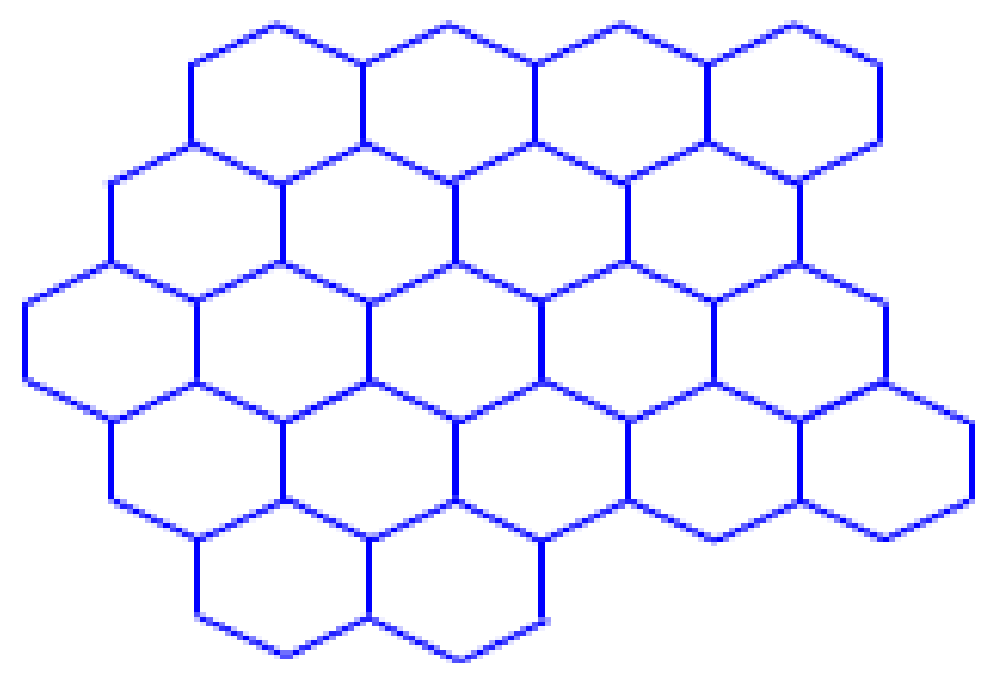

Figure 2. Example for Honeycomb graph

Case 2. When $n=10$.

Then the graph $B_{10}$ contains two cycles with one edge in common, which is isomorphic to the graph $H$, where $V(H)=V\left(C_{6}\right) \cup V\left(P_{4}\right)$ and $E(H)=E\left(C_{6}\right) \cup E\left(P_{4}\right) \cup e_{1} \cup e_{2}$, where $e_{1}$ and $e_{2}$ joins $\left(v_{1}, u_{1}\right)$ and $\left(v_{2}, u_{2}\right)$, where $v_{1}, v_{2} \in V\left(C_{6}\right), v_{1} \in N\left(v_{2}\right)$ and $u_{1}$ and $u_{2}$ are the end vetices of $P_{4}$. Hence $\gamma_{c s d}=\gamma_{c s d}\left(C_{6}\right)+\gamma_{c s d}\left(P_{4}\right)=3+2=\left\lceil\frac{n}{2}\right\rceil$.

Case 3. When $n \geq 10$.

In this case. the planar honey comb graph can be obtained by adding an edge that connects the end vertices of $m$ copies of either $P_{1}$ or $P_{2}$ or $P_{3}$ or $P_{4}$ with an adjacent vertices of $B_{10}$. By using case 2 and Theorem 3.9,

$$
\begin{aligned}
\gamma_{c s d} & =\gamma_{c s d}\left(B_{10}\right)+m \gamma_{c s d}\left(P_{1} \text { or } P_{2} \text { or } P_{3} \text { or } P_{4}\right) \\
& =5+\left\lceil\frac{n-10}{2}\right\rceil \\
& =5+\left\lceil\frac{n}{2}\right\rceil-5 \\
& =\left\lceil\frac{n}{2}\right\rceil .
\end{aligned}
$$

\section{Application}

Whenever we transfer a message from one mobile device which is in different signal range, to another mobile device which is in some other different signal range, then sometimes can be a loss of data or the message may be delivered after a long time. These are mainly due to the unstructured or unorganized way of locating message service systems and unsecured network. These problems can be solved using our complete secure domination. Through secure domination, we are providing the least or minimum number of message centers with which the entire block or chain of message centers can be covered and also secured. In this way, we propose the complete secure domination with minimum number of message centers to overcome the above mentioned issues. 


\section{References}

[1] M. Anderson, C. Barrientos, R. Brigham, J. Carrington, R. Vitray, J. Yellen, Maximum demand graphs for eternal security, J. Combin. Math. Combin. Comput. 61 (2007) 111-128.

[2] S. Benecke, Higher order domination of graphs, Master Thesis, University of Stellenbosch (2004).

[3] S. Benecke, E. J. Cockayne, C. M. Mynhardt, Secure total domination in graphs, Utilitas Math. 74 (2007) 247-259.

[4] F. Harary, Graph theory, Addison-Wesely, Reading Mass, 1st Edition (1969). 